\title{
GK Komplexe Mannigfaltigkeiten, Bayreuth
}

\section{von Thomas Peternell}

Das Graduiertenkolleg „Komplexe Mannigfaltigkeiten" an der Universität Bayreuth nahm am 1. Oktober 1990 als einer der ersten Kollegs seinen Betrieb auf. Mit seinen sechs Graduiertenstipendien, einer Postdoktorandenstelle und vier involvierten Hochschullehrern (H. Kerner, Th. Peternell, M. Schneider und F.O. Schreyer) ist es das kleinste aller Kollegs in Deutschland. Thematisch ist es, wie der Name schon andeutet, in der komplexen Analysis und komplexen algebraischen Geometrie, also im wesentlichen in einem Forschungsgebiet, angesiedelt. Auf der einen Seite bedeutet dies natürlich eine gewisse Spezialisierung, andererseits einen erheblichen Gewinn durch die Homogenität: alle Kollegiaten nehmen an allen Veranstaltungen des GKs teil; die Arbeitsgebiete sind noch nicht soweit voneinander entfernt, daß nicht jeder mit jedem über seine spezifischen Probleme reden kann. Das heißt natürlich auch, daß jeder Kollegiat sich an jeden der beteiligten Hochschullehrer wenden kann. In einem Fall hat es sogar eine direkte Doppelbetreuung einer Dissertation gegeben. Das Thema des GK hat viele Verbindungen zu anderen zentralen Gebieten der Mathematik, wie etwa der Topologie, der Differentialgeometrie und der Algebra.

Eine Besonderheit des Bayreuther Graduiertenkollegs sind die „institutionalisierten“ Sommerschulen, die jährlich stattfinden. Diese einwöchigen Kompaktseminare werden jeweils von zwei oder drei auswärtigen Referenten geleitet und werden von ca. jeweils 60-80 Teilnehmern besucht, von denen oft die Hälfte aus dem Ausland kommt. Die Referenten und Themen der letzten vier Jahre waren:

- J. Kollár, R. Lazarsfeld: Geometry of higherdimensional varieties

- M. Lübke, C. Okonek: Donaldson theory and complex geometry

- W. Fulton, P. Pragasz: Degeneracy loci and Schubert varieties

- V.I. Arnold, M.E. Kazarian: Singularity theory and symplectic geometry.

Durch die Sommerschulen, die häufigen Gastaufenthalte ausländischer Wissenschaftler in Bayreuth und die Reisemöglichkeiten zu europäischen Seminaren und Tagungen erhalten die Kollegiaten frühzeitig internationale Kontakte, insbesondere auch zu Doktoranden anderer Universitäten, und kommen in Berührung zu dem Wissenschaftsbetrieb auch außerhalb der eigenen Institution. Dies gab es früher nur in sehr begrenztem Umfang und ist nicht nur wissenschaftlich sehr fruchtbar, sondern wirkt natürlich auch motivierend.

Die erste Generation hat das Bayreuther Graduiertenkolleg um 1993 verlassen. Einige haben danach ein Jahr im Ausland verbracht; einer der Absolventen befindet sich auf dem halben Weg zur Habilitation, andere haben in der Industrie Erfolg. Die durchschnittliche Promotionsdauer betrug weniger als drei Jahre. Mehr als die Hälfte unserer Stipendiaten kommt von außerhalb, sie hätten vielleicht nicht alle ohne das Graduiertenkolleg promoviert. Wir haben nicht den Eindruck, daß das Promotionspotential in der Mathematik durch die traditionelle Promotionsintensität schon erschöpft ist. Angesichts der geringen Promotionsquote in der Mathematik ist dies auch nicht allzu verwunderlich. Andererseits mögen viele potentielle Promotionskandidaten durch die immer grösser werdenden Anforderungen an die Vorkenntnissen abgeschreckt werden. Durch gezielte Promotionsvorbereitung durch Spezial- und Steilkurse können Graduiertenkollegs hier Abhilfe schaffen. Natürlich können auch Diplomstudenten schon von GK's profitieren, etwa durch Spezialkurse. Die motivierende Wirkung auf Diplomstudenten ist sicherlich vorhanden; im letzten Jahr etwa schlossen 7 von 9 Diplomanden in der algebraischen Geometrie in Bayreuth das Diplom bis zum 10. Semester ab, zum Teil inklusive eines Auslandsjahres. Es ist allerdings noch nicht zu erkennen, ob sich das Mathematikstudium durch die Graduiertenkollegs auf Dauer wesentlich ändert. Denn der „normale“ Student im 5. oder 6 . Semester wird wohl ein Graduiertenkolleg nicht als Perspektive sehen. Eine solide wissenschaftliche Ausbildung und Diplomarbeit ist andererseits unabdingbare Voraussetzung für die Aufnahme in das GK; rein rezeptive Aufnahme eines Stoffes ist eben etwas ganz anderes als das selbständige wissenschaftliche Arbeiten, das ja auch ansatzweise bei einer guten Diplomarbeit vorhanden sein sollte. Wie sollte man sonst die Promotionsfähigkeit feststellen?

Fazit: Wir haben durchweg positive Erfahrungen mit dem GK gemacht. Ein GK erzeugt eine kritische Masse, um Promotionsstudien zu intensivieren und $\mathrm{zu}$ beschleunigen, verschafft den Stipendiaten internationale Kontakte und erleichtert es durch die Integration in eine "Forschergruppe", die eigene Arbeit in größere Zusammenhänge einzuordnen.

Adresse des Autors:

Prof. Dr. Thomas Peternell

Mathematisches Institut

Universität Bayreuth

95440 Bayreuth 